\title{
Association between the expression of core 3 synthase and survival outcomes of patients with cholangiocarcinoma
}

\author{
PATCHARAPORN BOOTTANUN ${ }^{1-3}$, YOSHINORI INO $^{3}$, KAZUAKI SHIMADA $^{4}$, \\ NOBUYOSHI HIRAOKA ${ }^{3,5}$, KIYOHIKO ANGATA ${ }^{2}$ and HISASHI NARIMATSU ${ }^{1,2}$ \\ ${ }^{1}$ Graduate School of Comprehensive Human Sciences, Major in Medical Sciences, \\ Clinical Sciences Program, Faculty of Medicine, University of Tsukuba, Tsukuba, Ibaraki 305-8575; \\ ${ }^{2}$ Molecular and Cellular Glycoproteomics Research Group, Department of Life Science and Biotechnology, \\ Cellular and Molecular Biotechnology Research Institute, National Institute of Advanced Industrial Science and Technology, \\ Tsukuba, Ibaraki 305-8565; ${ }^{3}$ Division of Molecular Pathology, National Cancer Center Research Institute; \\ ${ }^{4}$ Hepatobiliary and Pancreatic Surgery Division, National Cancer Center Hospital; ${ }^{5}$ Department of Analytical Pathology, \\ National Cancer Center Research Institute, Chuo-ku, Tokyo 104-0045, Japan
}

Received May 12, 2021; Accepted August 4, 2021

DOI: $10.3892 / \mathrm{ol} .2021 .13021$

\begin{abstract}
Cholangiocarcinoma (CCA) is a highly aggressive and metastatic type of malignant carcinoma that is associated with high mortality rates and is difficult to detect at early stages. Core 3 structure is a mucin-type $O$-glycans synthesized by $\beta 1,3-N$-acetylglucosaminyltransferase 6 (core 3 synthase), which plays an important role in the digestive system, in particular gastrointestinal goblet cells. It has been reported that core 3 synthase-expressing cells show lower migratory and invasive rates, and lower metastatic activity. A immunohistochemical study also showed that this enzyme was expressed in normal epithelial cells of the colon, but completely disappeared in colorectal cancer cells. The present study aimed to identify biomarkers that could be used to predict the prognosis of patients with CCA. Pathological
\end{abstract}

Correspondence to: Professor Hisashi Narimatsu, Molecular and Cellular Glycoproteomics Research Group, Department of Life Science and Biotechnology, Cellular and Molecular Biotechnology Research Institute, National Institute of Advanced Industrial Science and Technology, 1-1-1 Higashi, Tsukuba, Ibaraki 305-8565, Japan E-mail: yuyoshik194@gmail.com

Dr Nobuyoshi Hiraoka, Division of Molecular Pathology, National Cancer Center Research Institute, 5-1-1 Tsukiji, Chuo-ku, Tokyo 104-0045, Japan

E-mail: nhiraoka@ncc.go.jp

Abbreviations: 6-sulfo LacNAc, 6-sulfated $N$-acetyllactosamine; B3GNT6, $\beta 1,3-N$-acetylglucosaminyltransferase 6 ; CCA, cholangiocarcinoma; dCCA, distal cholangiocarcinoma; eCCA, extrahepatic cholangiocarcinoma; iCCA, intrahepatic cholangiocarcinoma; LacNAc, $N$-acetyllactosamine; pCCA, perihilar cholangiocarcinoma

Key words: $\beta 1,3-N$-acetylglucosaminyltransferase 6 , core 3 synthase, immunohistochemistry, biomarker, cholangiocarcinoma specimens of 185 CCA tissues were immunohistochemically stained with two antibodies, G8-144 and MECA-79, which recognize core 3 synthase and 6 -sulfated $N$-acetyllactosamine on the extended core-1 $O$-glycans, respectively. The association between G8-144 or MECA-79 positivity and patient prognosis was statistically analyzed. Positive expression of G8-144 was associated with improved prognosis in patients with distal CCA (dCCA). Patients with dCCA positive for G8-144 showed lower mortality rates than those with negative expression. However, the positive expression of MECA-79 was associated with CCA progression and metastasis, indicating that it is a poor prognostic marker for CCA. In conclusion, as both antibodies resulted in mirror-image staining, the involvement of G8-144 and MECA-79 in $O$-glycan synthesis could be considered as potential favorable and unfavorable biomarkers, respectively, for CCA prognosis.

\section{Introduction}

Cholangiocarcinoma (CCA) or bile duct cancer is a serious health problem worldwide, especially in Asian countries (1), and its incidence is increasing. Owing to the difficulty associated with achieving early-stage diagnosis, CCA has a high mortality rate. CCA has been classified into two types based on its anatomical location: intrahepatic CCA (iCCA) and extrahepatic CCA (eCCA) $(1,2)$. It has been reported that these have different carcinogenic pathways (3). According to the anatomical site, further eCCA is divided into two categories, distal (dCCA) type and perihilar (pCCA) type, also known as Klatskin tumors (4). In CCA, the dCCA is expected to have the highest survival rates, followed by the pCCA and iCCA types. Thailand has the highest incidence of CCA (1). The incidence rate of CCA has been decreasing in Thailand in the past 10 years; however, the death of patients with CCA is still higher than six per 100,000 people (5). In Japan, the mortality rate of CCA is 5.85 in total deaths per 100,000 (6). Currently, serum levels of carcinoembryonic antigen (CEA) 
and CA19-9 have been used in the diagnosis and prognosis of CCA. However, the sensitivity and specificity are insufficient for the early diagnosis of CCA. Therefore, serum tests must be coupled with imaging methods, such as ultrasound, computed tomography (CT), and magnetic resonance imaging (MRI) methods, to achieve a better diagnosis (7). Currently surgical resection is only curative treatment. Thus, we need to understand CCA more profoundly to ameliorate the prognosis of patients with CCA.

The mucin-type $O$-glycans attached to the serine and threonine residues of proteins are diverse in structure and function. In fact, alterations in various mucin-type $O$-glycan structures have been reported during tumor progression $(6,8)$. As shown in Fig. 1, $O$-glycans are classified into several core structures that are specifically synthesized by unique enzymes (9). Core 3 structure is a mucin-type $O$-glycan that plays important roles in differentiation and mucosal barrier formation in digestive organs, including gastrointestinal goblet cells. The core 3 structure is synthesized by $\beta 1,3-N$-acetylglucosam inyltransferase 6 (B3GNT6 or core 3 synthase), which adds GlcNAc with $\beta 1,3$-linkage to the Tn antigen (GalNAc $\alpha$-serine/ threonine) $(10,11)$. Because the same 3'position of GalNAc in the Tn antigen is utilized by core 1 synthase, core 3 synthesis may compete with core 1 synthesis, as shown in Fig. 1.

A monoclonal antibody against core 3 synthase, G8-144, was established in a previous study (10). Immunohistochemical analysis of colorectal cancer tissues from familial adenomatous polyposis patients demonstrated that the enzyme was expressed in the normal epithelial cells of colorectal tissues, mainly in goblet cells, but decreased or disappeared in cancer cells (10). Moreover, compared to parental cells, the cells expressing core 3 via transfection of the core 3 synthase gene (B3GNT6 gene) showed lower migration activity in vitro as well as very low metastatic activity when core 3-expressing cells were introduced into nude mice (10). Core 3-expressing prostate cancer cells were also found to decrease the activity of tumor formation and metastatic activity to the lymph nodes by attenuating the maturation and heterodimerization of integrin $\alpha 2 \beta 1$ (12). The core 3 structure was recently reported to be involved in EMT-MET regulation via the MUC1/p53/miR-200c signaling cascade (13).

$O$-glycan pathways have been suggested to be correlated with cancer progression (14). For instance, Tn antigen was identified to be increased in colon cancer and breast cancer (15-17), and core 1 and core 2 structures are often found in breast and prostate cancers $(18,19)$. However, antibodies specifically recognizing the core structures of $O$-glycans have not been established; this might be due to modification of core $O$-glycans by additional glycosylation, such as fucosylation and sialylation. For example, the 6 -sulfated $N$-acetyllactosamine (6-sulfo LacNAc) structure, which is synthesized on the extended core $1 O$-glycans, is known as the epitope of the MECA-79 antibody (Fig. 1) (20).

In the present study, we aimed to investigate the clinicopathological correlation of core 3 synthase expression with the prognosis of patients with eCCA relative to that with MECA-79 expression. Briefly, an immunohistochemical study was conducted with 185 patients with eCCA using two antibodies, G8-144 (anti-core 3 synthase) and MECA-79 (anti-6-sulfo LacNAc on core 1). As a result, these $O$-glycosylation-related antibodies, G8-144 and MECA-79, were identified to be associated with eCCA survival rates in an opposing manner.

\section{Materials and methods}

Cholangiocarcinoma specimens. This study was approved by the Institutional Review Board of the National Cancer Center, Tokyo, Japan (2019-0186). Informed consent was obtained from all participants before their participation in the study. All clinical investigations were conducted in accordance with the principles of the Declaration of Helsinki.

This study included 185 patients, 118 with dCCA and 67 with pCCA underwent surgical resection between 2006 and 2016 at the National Cancer Center Hospital, Tokyo. All patients included in this study underwent macroscopic curative resection. None of the patients received radiotherapy or chemotherapy before surgery. Recurrence was diagnosed when a new local or distant metastatic lesion was detected in imaging studies or when an increase in tumor marker levels with deterioration of the patient's condition was observed. All of the CCA were diagnosed according to the World Health Organization (WHO) classification (2), the 8th UICC/AJCC TNM Classification $(3,4)$. Macroscopic types of eCCA and the following histopathological variables were evaluated following the Japanese Society of Biliary Surgery (JSBS) classification (4): lymphatic, venous, and perineural invasions. The clinicopathological characteristics of patients are summarized in Table SI. All patients with stage IV disease were diagnosed on the basis of para-aortic lymph node involvement.

Preparation of the two antibodies, G8-144 (anti-core 3 synthase) and MECA-79 (anti-6-sulfo LacNAc on core 1). The monoclonal antibody that reacts with core 3 synthase, called G8-144, was previously established by our laboratory (10). First, G8-144 was purified using protein-G beads. G8-144 secreted from hybridoma cells was diluted with PBS to a total volume of $25 \mathrm{ml}$ and applied to $200 \mu \mathrm{l}$ protein-G Sepharose fast flow (GE Healthcare) for antibody capture at $4^{\circ} \mathrm{C}$ overnight. The protein-G beads were washed and eluted with $0.1 \mathrm{M}$ glycine ( $\mathrm{pH} 2.5$ ), followed by neutralization with $3 \mathrm{M}$ Tris- $\mathrm{HCl}$ (pH 8.5). Subsequently, the purified antibody was dialyzed with pH 7.5 phosphate buffer saline (PBS; pH; Fujifilm Wako Pure Chemical Corporation), and concentrated using an Amicon ultra $0.5 \mathrm{ml}$ filter cut off $100 \mathrm{~K}$ (Merck). Purified G8-144 was subjected to immunohistochemical analysis.

The MECA-79 antibody recognizes a sulfated $\mathrm{N}$-acetyllactosamine on extended core 1 mucin-type $O$-glycan, $\mathrm{Gal} \beta 1 \rightarrow 4$ (sulfo $\rightarrow 6$ ) GlcNAc $\beta 1 \rightarrow 3 \mathrm{Gal} \beta 1 \rightarrow 3$ GalNAc $(6$-sulfo LacNAc on core 1) (20). The MECA-79 rat monoclonal IgM antibody used to stain the serial pathological sections was purchased from BD Biosciences.

Construction of core 3 synthase expression plasmid DNA and western blotting. To confirm the specificity of G8-144 before performing the immunohistochemical study, the purified G8-144 antibody was subjected to western blot analysis. As most of the core 3 synthase-expressing cells, which were transfected with core 3 synthase cDNA, were reported as overexpressing cell lines, there are only a few cell lines that stably express core 3 synthase (21). To prepare core 3 


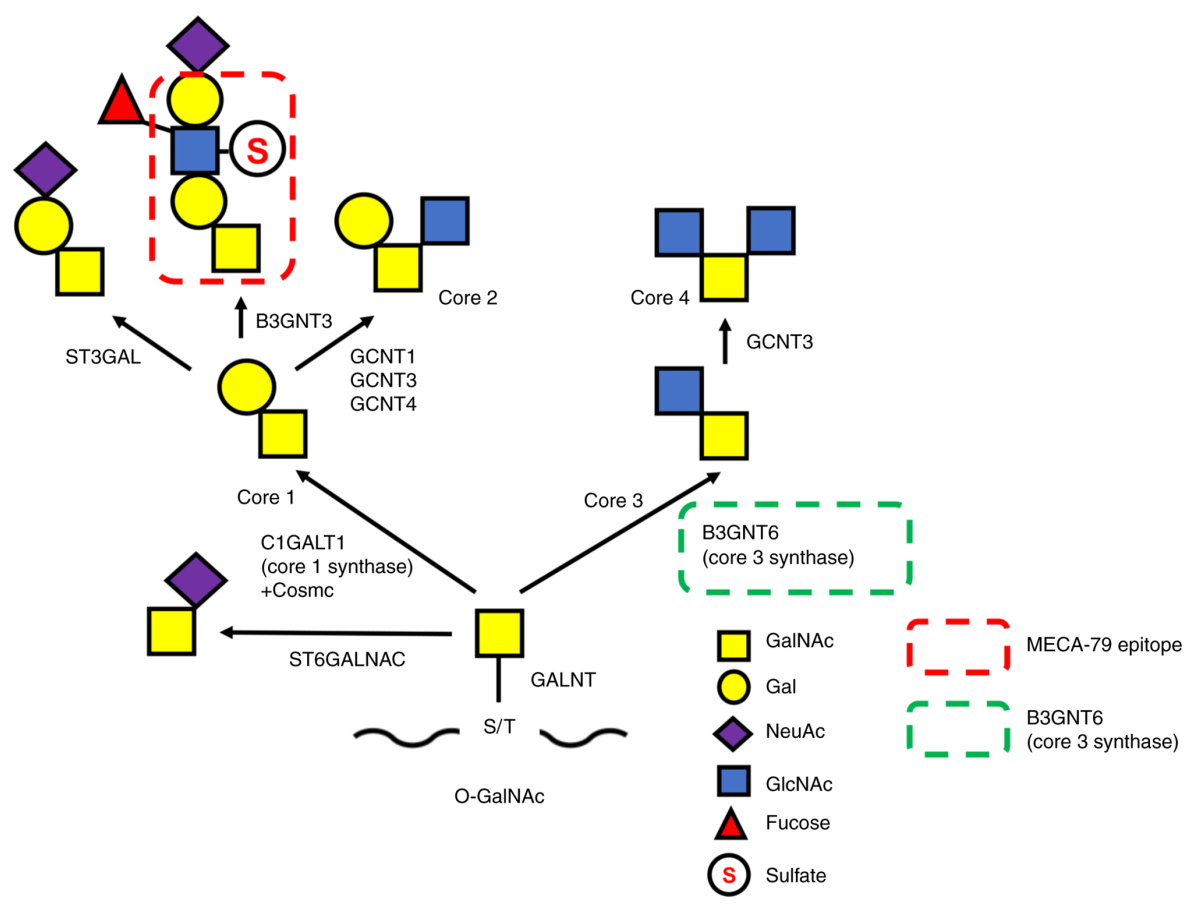

Figure 1. $O$-glycan synthetic pathway. Mucin $O$-glycosylation is initiated by the transfer of GalNAc from UDP-GalNAc to $\mathrm{S}$ or T residues. This process is catalyzed by multiple GALNTs resulting in the generation of the Tn antigen. Thereafter, the Tn antigen is extended by specific enzymes, such as C1GALT1/core 1 synthase with its chaperone Cosmc or B3GNT6/core 3 synthase, to form the structure of core 1 or core 3, respectively. The MECA-79 epitope (a sulfated $\mathrm{N}$-acetyllactosamine on extended core 1) is synthesized on the structure of core 1. Core 3 synthase and the MECA-79 epitope are indicated by green and red dashed line rectangles, respectively. GalNAc, $N$-acetylgalactosamine; S, serine; T, threonine; GALNTs, polypeptide- $N$-acetyl-galactosaminyltransferases; B3GNT6, $\beta 1,3-N$-acetylglucosaminyltransferase 6; C1GALT1, glycoprotein- $N$-acetylgalactosamine 3 $\beta$-galactosyltransferase 1 ; ST3GAL, ST3 $\beta$-galactoside a-2,3-sialytransferase 3; GCNT, glucosaminyl ( $N$-acetyl) transferase; ST6GALNAC, ST6 $N$-acetylgalactosaminide $\alpha$-2,6-sialyltransferase; $O$-GalNAc, $O$-linked $N$-acetylgalactosamine; NeuAc, $N$-acetylneuraminic acid.

synthase (B3GNT6), B3GNT6 cDNA was subcloned into the pCDN3.1 vector (Thermo Fisher Scientific, Inc.), resulting in pcDNA3.1-B3GNT6. Thereafter, the purified plasmid DNA was transfected into HEK293T cells using Lipofectamine ${ }^{\circledR}$ LTX DNA transfection reagent (Thermo Fisher Scientific, Inc.), according to the manufacturer's protocol. Forty-eight hours after transfection, the cells transiently expressing the core 3 synthase were collected and lysed with lysis buffer containing $1 \%$ NP-40, 0.1\% SDS, $50 \mathrm{mM}$ Tris-Cl pH 7.4, $150 \mathrm{mM} \mathrm{NaCl}$, $5 \mathrm{mM}$ EDTA, $1 \mathrm{mM}$ PMSF, and protease inhibitors (Merck). Frozen tissues were obtained from resected surgical specimens and were stored at $-80^{\circ} \mathrm{C}$ until use. For comparison between pathological specimens and cancer cell lines, frozen CCA tissue samples from the G8-144 positive cases and HAL8 cells, a lung adenocarcinoma cell line (22), were lysed using the lysis buffer. After centrifugation, the supernatant was collected, and the protein concentration was measured using a micro-BCA kit (Thermo Fisher Scientific, Inc.). Core 3 synthase might be expressed in minute amounts, so collecting them from the positive sites in the frozen tissue may provide limited yield. Thus, the samples were concentrated from frozen tissue and HAL8 cells using an Amicon $3 \mathrm{~K}$ (Merck) tube. The proteins were then loaded and separated using 5-20\% gradient SDS-PAGE, while the HEK235T+B3GNT6 and HEK293T cell lysates were loaded at $5 \mu \mathrm{g} / \mu \mathrm{l}$ and blotted onto a polyvinylidene fluoride membrane (Bio-Rad). Proteins that reacted with G8-144 or GAPDH (Fujifilm Wako) were detected using anti-mouse IgG conjugated with HRP (Thermo Fisher Scientific, Inc.) and Immobilon Forte western HRP substrate (Merck).
Immunohistochemistry. The representative tumor tissue was used for all the examinations of the tissue specimen in this study. To evaluate the expression levels of core 3 synthase and 6-sulfo LacNAc on core 1 in CCA, serial CCA sections were immunohistochemically stained using G8-144 and MECA-79. Briefly, formalin-fixed and paraffin-embedded sections were deparaffinized in xylene and rehydrated using a graded ethanol series. After the sections were washed with deionized water, endogenous peroxidase was blocked with $0.3 \% \mathrm{H}_{2} \mathrm{O}_{2}$ in methanol for $20 \mathrm{~min}$. Antigens were retrieved via autoclaving in $1 \mathrm{X}$ Envision ${ }^{\mathrm{TM}}$ flex target retrieval solution at high $\mathrm{pH}$ (Dako). The sections were washed with PBS, and endogenous biotin and biotin-binding factors were blocked with a biotin-blocking system (Dako). After washing with PBS, the sections were incubated with each antibody overnight at $4^{\circ} \mathrm{C}(23)$. G8-144 (anti-core 3 synthase antibody) was used at a concentration of $0.5 \mu \mathrm{g} / \mathrm{ml}$ while MECA-79 (anti-6-sulfo LacNAc on core 1 antibody) was used at a concentration of $1.0 \mu \mathrm{g} / \mathrm{ml}$. The sections were incubated with the secondary antibody, biotinylated goat anti-mouse IgG (Vector Laboratories) for G8-144 or biotinylated goat anti-rat IgM (Vector Laboratories) for MECA-79 for $30 \mathrm{~min}$. Thereafter, they were reacted for $30 \mathrm{~min}$ to form the avidin-biotin-peroxidase complex (Vectastain ABC kitl Vector Laboratories). Staining was visualized with diaminobenzidine. The sections were also counterstained with hematoxylin.

Cancerous tissues were divided into two regions: non-invasive and invasive regions. Cancer cells that remained on the epithelial layer of the bile duct were determined to be 
Table I. Summary of core 3 synthase and MECA-79 antibody staining of the dCCA specimens.

A, Core 3 synthase

\begin{tabular}{|c|c|c|c|c|c|c|c|}
\hline \multirow[b]{2}{*}{$\mathrm{dCCA}$} & \multicolumn{4}{|c|}{ Expression (non-invasive/invasive) } & \multirow{2}{*}{$\begin{array}{c}\text { Total positive } \\
\text { non-invasive, n }(\%)\end{array}$} & \multirow{2}{*}{$\begin{array}{l}\text { Total positive } \\
\text { invasive, n }(\%)\end{array}$} & \multirow{2}{*}{$\begin{array}{c}\text { Total positive, } \\
\mathrm{n}(\%)\end{array}$} \\
\hline & $+/+$ & $+/-$ & $-/+$ & $-/-$ & & & \\
\hline Cases, $\mathrm{n}$ & 31 & 28 & 6 & 53 & $59(50.00)$ & $37(31.41)$ & $65(55.10)$ \\
\hline
\end{tabular}

B, MECA-79 staining

\begin{tabular}{|c|c|c|c|c|c|c|c|}
\hline \multirow[b]{2}{*}{$\mathrm{dCCA}$} & \multicolumn{4}{|c|}{ Expression (non-invasive/invasive) } & \multirow{2}{*}{$\begin{array}{l}\text { Total positive } \\
\text { non-invasive, } \mathrm{n}(\%)\end{array}$} & \multirow{2}{*}{$\begin{array}{l}\text { Total positive } \\
\text { invasive, } \mathrm{n}(\%)\end{array}$} & \multirow{2}{*}{$\begin{array}{c}\text { Total positive, } \\
\mathrm{n}(\%)\end{array}$} \\
\hline & $+/+$ & $+/-$ & $-/+$ & $-/-$ & & & \\
\hline Cases, $\mathrm{n}$ & 42 & 13 & 27 & 36 & $55(46.61)$ & $69(58.47)$ & $82(69.49)$ \\
\hline
\end{tabular}

+ , positive expression; -, negative expression; dCCA, distal cholangiocarcinoma.

non-invasive cancer cells, while cells infiltrating beyond the epithelial layer were determined to be invasive cancer cells. To evaluate the level of staining positivity, sections were quantitatively scored based on the percentage of positive cells in the total cancer cells (1-100\%). When the immunolabelled positive cells were more than $1 \%$, the cancer was judged as a positively stained. The staining scores were evaluated by two independent pathologists that were blinded to the clinical status.

Statistical analysis. The associations between characteristic variables were analyzed by chi-square or the Fisher exact tests. Postoperative overall survival (OS), disease-free survival (DFS) rates, and the expression of core 3 synthase and MECA-79 positivity were calculated using the Kaplan-Meier method and analyzed by the log-rank test (24). Factors found to be significant in univariate analysis were incorporated into the multivariate analysis using the Cox proportional hazards model (backward elimination method). Differences were considered statistically significant at $\mathrm{P}<0.05$, and all statistical analyses were performed using StatView-J 5.0 software (Abacus Concepts).

\section{Results}

Confirmation of the specificity of the G8-144 antibody using CCA tissue. To determine whether G8-144 could be applied to immunohistochemical studies for diagnostic purposes, we assessed the specificity of G8-144 by western blotting using CCA samples. HAL8, a lung adenocarcinoma cell line, was used as a positive control cell line expressing core 3 synthase [(22), data not shown]. HEK293T cells that did not express core 3 synthase were compared with HEK293T cells transfected with B3GNT6 cDNA. Lysates purified from CCA tissues that were positive for G8-144 staining were blotted. The results indicated that this enzyme was approximately 45 to $50 \mathrm{kDa}$, as detected using the G8-144 antibody (Fig. S1, upper right). The size differences may be due to differences in glycosylation between transient expression in cultured cells and human CCA tissue. Western blotting results showed several weak positive bands with higher molecular weights, which may be polymers or complexes with other proteins. Such findings indicate that a detectable amount of core 3 synthase with G8-144 antibody was expressed in the CCA tissue, unlike that in colorectal cancer (11).

Core 3 synthase expression in CCA tissues as revealed by immunohistochemical analysis with the G8-144 antibody. To explore the expression of core 3 synthase in CCA and its clinicopathological correlation with patient prognosis, an immunohistochemical study using the G8-144 antibody was performed using $185 \mathrm{CCA}$ patient specimens. Briefly, we classified the $185 \mathrm{CCA}$ pathological specimens into two groups, 118 samples of dCCA and 67 of pCCA, based on their anatomical location. The dCCA specimens were retrieved from 98 males and 20 females of different ages; median age was 72 years. As shown in (Table SI), the study included 64 cases of patients $\geq 72$ and 54 cases of patients $<72$ years of age. Meanwhile, 51 male and 16 female samples were retrieved for pCCA; median age was 67 years. There were 34 cases of patients $\geq 67$ years and 33 cases of patients $<67$ years).

The results of immunohistochemical staining with G8-144 showed that dCCA and pCCA were positive for G8-144 in 55.1 and $59.7 \%$ of the total cases, respectively (Table I). No core 3 synthase was expressed in any of the normal epithelial cells in the bile duct (Fig. S1, lower left). It was expressed frequently in the intraepithelial non-invasive cancer cells (Fig. 2, upper left panel) and the invasive cancer cells (Fig. 2, upper right panel) at the shallow invasive lesion, that is, near mucosal layer in the bile duct wall in the eCCA. It could be found in a low frequency that cancer cells invaded deeply into the bile duct expressed core 3 synthase. These findings were observed in both of dCCA and pCCA. The frequencies of core 3 synthase expression in non-invasive cancer cells and invasive cancer cells were 52.7 and $33.6 \%$ in dCCA, respectively (Table I), and 51.9 and $38.2 \%$ in pCCA, respectively (data not shown). 


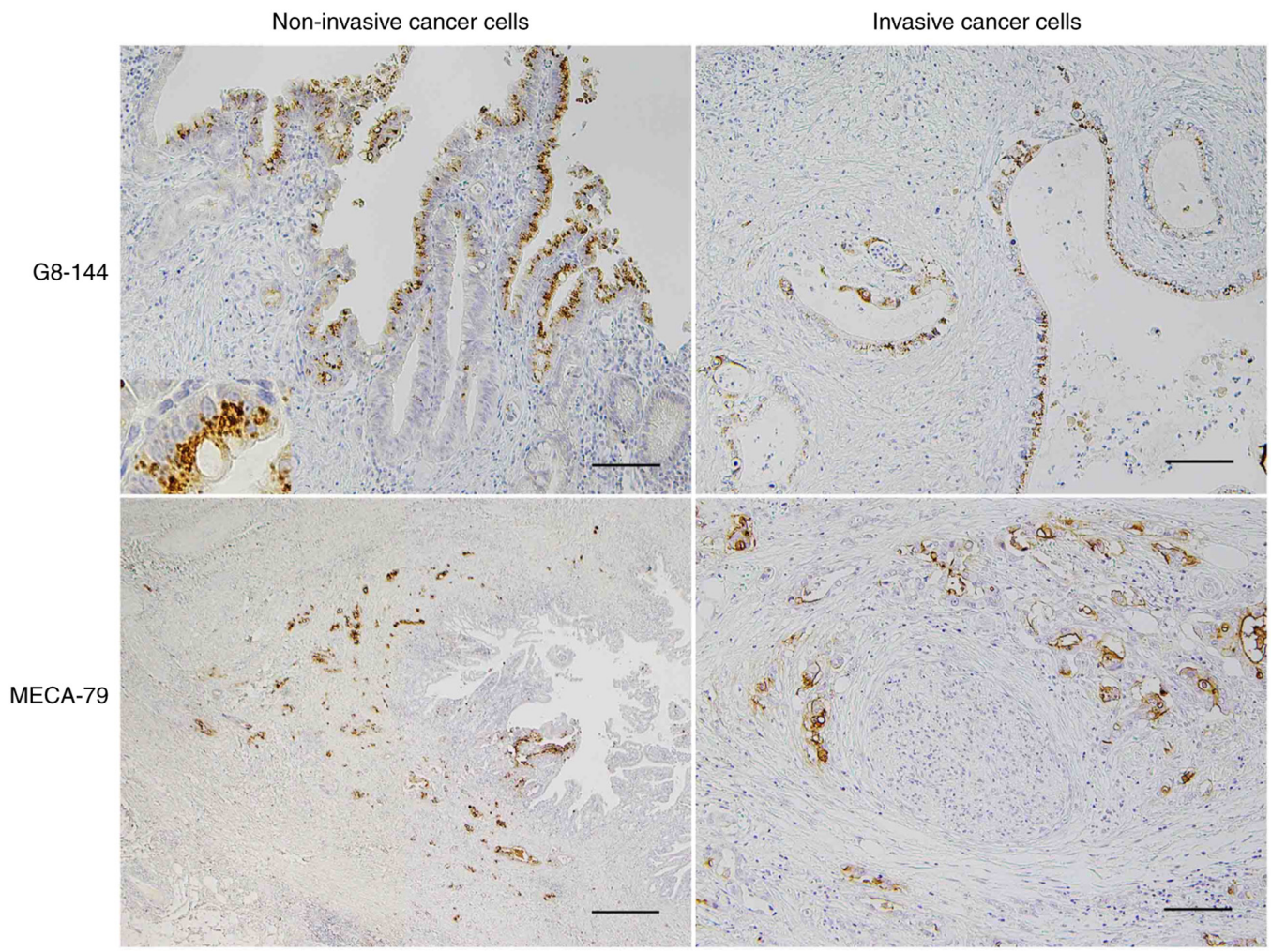

Figure 2. Immunohistochemical detection of $\beta 1,3-N$-acetylglucosaminyltransferase 6/core 3 synthase using G8-144 and MECA-79 antigen in CCA. Core 3 synthase was immunohistochemically detected using the G8-144 antibody in dCCA (upper sections). Core 3 synthase was mainly expressed in the intraepithelial non-invasive cancer cells and some cancer cells at shallow invasive areas, but not in any normal epithelial cells in the bile duct. Proliferating dCCA cancer cells forming a papillary structure toward the luminal side (upper direction) in the surface epithelial layer expressed core 3 synthase (upper left). Core 3 synthase was observed as an intracytoplasmic dot-like feature (upper left, inset) that was consistent with the presence of core 3 synthase in the Golgi apparatus. Also, core 3 synthase was not frequently present in the invasive cancer cells (upper right). Scale bar (upper sections), $100 \mu \mathrm{m}$. MECA-79 antigen was detected in dCCA cancer cells in both non-invasive and invasive areas, but it was predominantly expressed in deeply invasive cancer cells (lower sections). In the deeply infiltrating dCCA with a bile duct lumen at the right in the panel, cancer cells expressing MECA-79 antigen were observed mostly in the invaded area and the intraepithelial non-invasive area (lower left). MECA-79-positive cancer cells, which are moderately to poorly differentiated adenocarcinoma with an aborted gland structure or nest formation morphology, showed perineural invasion at the invasive front (lower right). Scale bar (lower left), $500 \mu \mathrm{m}$ and scale bar (lower right), $50 \mu \mathrm{m}$. CCA, cholangiocarcinoma; dCCA, distal cholangiocarcinoma.

Core 1 structure with the MECA-79 epitope identified on epithelial cells of the bile duct and CCA. To obtain further knowledge on $O$-glycan expression in eCCA, MECA-79, which is specific to 6-sulfated $\mathrm{N}$-acetyllactosamine (6-sulfo LacNAc) on the extended core-1 $O$-glycans (Fig. 1), was compared to G8-144. Serial sections of the pathological specimen used for G8-144 staining were subjected to MECA-79 staining (Fig. 2). In contrast to G8-144 staining, MECA-79 antigen was expressed in the non-cancerous epithelial cells of the bile duct (Fig. S1, lower right). MECA-79 antigen was expressed in 69.5 and $55.2 \%$ in dCCA and pCCA, respectively. It tended that MECA-79 antigen was expressed more frequently in deeply infiltrating cancer cells (Fig. 2, lower left panel) and often found in cancer cells showing lymphovascular or perineural invasion (Fig. 2, lower right panel). The frequencies of MECA-79 antigen expression in non-invasive cancer cells and invasive cancer cells were 49.1 and $62.7 \%$ in dCCA, respectively (Table I), and 42.3 and $50.9 \%$ in pCCA, respectively (data not shown). Thus, cancer cells expressing core 3 synthase and those expressing MECA-79 were different cancer areas. Furthermore, we often observed that in the serial specimens, cancer cells expressing core 3 synthase did not display MECA-79-positivity in the non-invasive (Fig. 3, upper left and right) or invasive (Fig. 3, lower left and right) areas.
Correlation of G8-144 positivity with the prognosis of dCCA patients. As summarized in Table I, the 118 dCCA patients were divided into four groups: i) positive staining in both non-invasive and invasive regions (31 patients), ii) positive in non-invasive but negative in invasive region (28 patients), iii) negative in non-invasive but positive in invasive region (6 patients), and iv) negative in both non-invasive and invasive regions (53 patients). pCCA patients were not included in this table as no correlation was found between G8-144 staining and patient prognosis as shown in later.

To analyze the correlation between core 3 synthase expression and the survival rate of dCCA patients, the classified staining results were subjected to Kaplan-Meier survival analysis (Fig. 4). As shown in the left panels of Fig. 4, the dCCA patients showing positive G8-144-staining in non-invasive or invasive cancer regions (65 of the 118 patients, $55.1 \%$ ) had a significantly better prognosis than the G8-144-negative patients (53 patients). The difference between core 3 synthase -positive and -negative survival rates of dCCA patients was significant in postoperative OS (upper panels) and DFS (lower panels) ( $\mathrm{P}=0.007$ and $\mathrm{P}=0.024$, respectively). The dCCA patients with positive staining in the non-invasive region displayed a better prognosis than those with negative $\operatorname{dCCA}(\mathrm{P}=0.031$ and $\mathrm{P}=0.035$ 


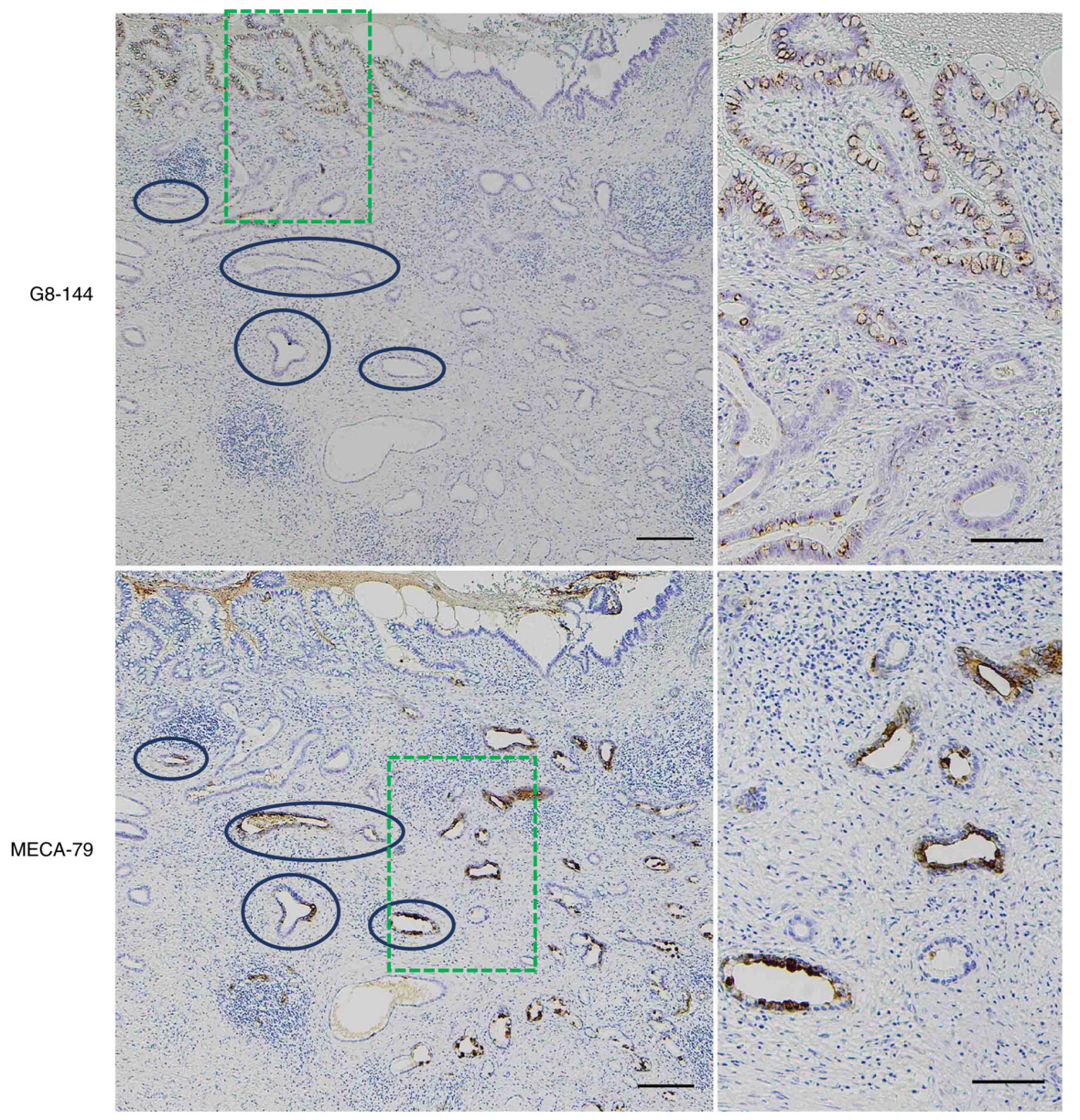

Figure 3. G8-144-positive cancer cells and MECA-79-positive cancer cells are differently distributed. A mirror-image stain was observed between G8-144 and MECA-79. These images are a representative example of distal cholangiocarcinoma. Cancer cells expressing core 3 synthase (G8-144-positive cells) were mostly found in the luminal epithelial layer and next to the shallow invasive area (upper left), where bile duct lumen was present in the upper portion in the panel. Core 3 synthase-positive cancer cells often show abundant intracytoplasmic mucin and look similar to goblet cells, as shown in the upper right panel. By contrast, expression of MECA-79 antigen was observed in the deeply invading cancer cells (lower left). MECA-79 antigen was expressed in the cytoplasmic and luminal surface of the cancer glands in invasive adenocarcinoma cells (lower right). The distribution of core 3 synthase-positive cells and MECA-79 antigen-positive cells showed mirror-image staining. The dotted line areas are enlarged and shown in the right panels. Scale bar (upper sections), $200 \mu \mathrm{m}$ and scale bar (lower sections), $100 \mu \mathrm{m}$.
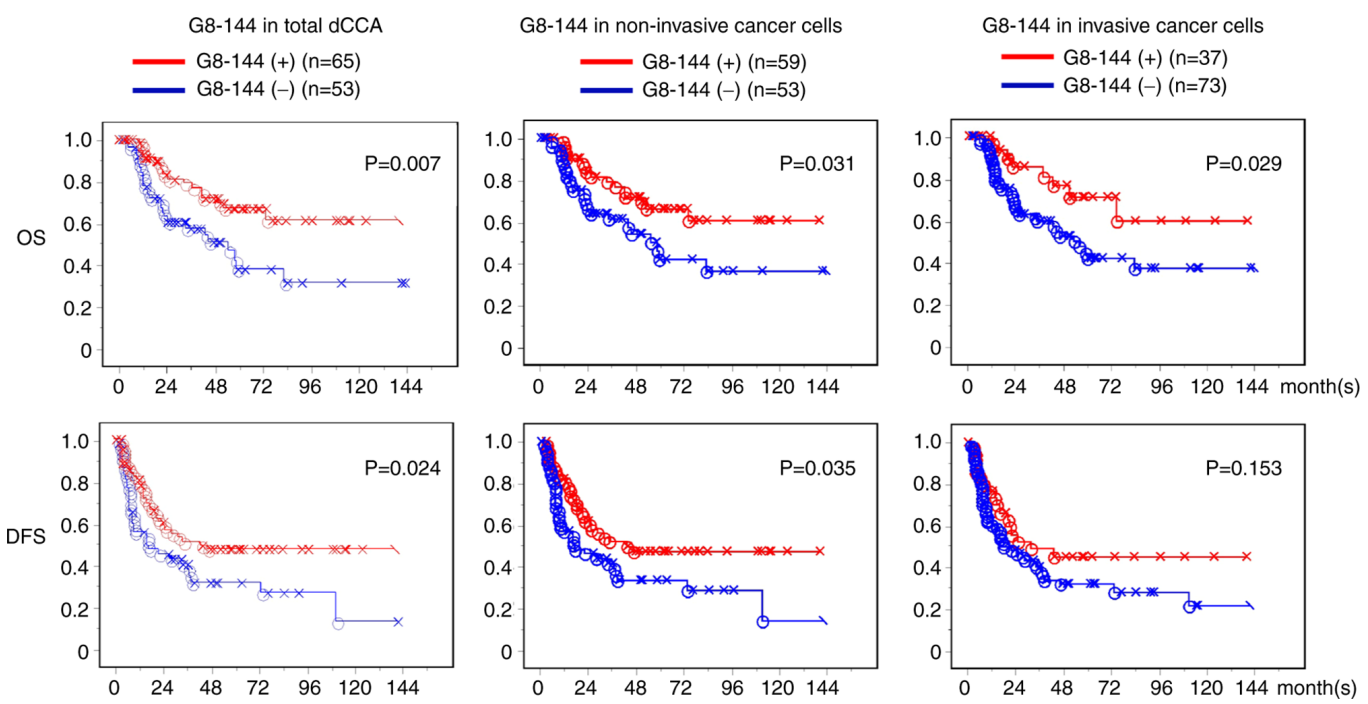

Figure 4. Survival rates of patients with dCCA with $\beta 1,3-\mathrm{N}$-acetylglucosaminyltransferase 6/core 3 synthase expression. Kaplan-Meier survival curves were plotted for patients with G8-144(+) (red) or G8-144(-) (blue) dCCA. The expression level of core 3 synthase, as indicated by G8-144 expression, was significantly associated with the longer survival of patients after tumor resection (month). Postoperative OS rates are presented in the upper panel and DFS rates are presented in the lower panels. Total dCCA (left panels), non-invasive cancer cells (center panels) and invasive cancer cells (right panels). dCCA, distal cholangiocarcinoma; OS, overall survival; DFS, disease-free survival; (+), positive; (-), negative. 
Table II. Univariate and multivariate analyses of prognostic factors in patients with distal cholangiocarcinoma $(\mathrm{n}=118)$.

A, Disease-free survival

\begin{tabular}{|c|c|c|c|c|}
\hline \multirow[b]{2}{*}{ Variables } & \multicolumn{2}{|c|}{ Univariate analysis } & \multicolumn{2}{|c|}{ Multivariate analysis } \\
\hline & $\mathrm{HR}(95 \% \mathrm{CI})$ & P-value & $\operatorname{HR}(95 \% \mathrm{CI})$ & P-value \\
\hline Age, $\leq 72$ years vs. $>72$ years & $0.905(0.555-1.477)$ & 0.6900 & & \\
\hline Sex, female/male & $0.565(0.312-1.024)$ & 0.0600 & & \\
\hline Pathological tumor status, Tis+T1+T2 vs. T3 & $1.911(0.970-3.765)$ & 0.0610 & & \\
\hline Pathological node status, N0 vs. N1+N2 & $2.598(1.566-4.311)$ & $0.0002^{\mathrm{a}}$ & $2.046(1.208-3.466)$ & $0.0080^{\mathrm{a}}$ \\
\hline Pathological metastatic status, M0 vs. M1 & $2.574(1.163-5.698)$ & $0.0200^{\mathrm{a}}$ & & \\
\hline Histological grade, G1 vs. G2-4 & $0.901(0.458-1.775)$ & 0.7640 & & \\
\hline Tumor margin status, negative vs. positive & $2.518(1.540-4.115)$ & $0.0002^{\mathrm{a}}$ & $2.296(1.399-3.768)$ & $0.0010^{\mathrm{a}}$ \\
\hline Lymphatic invasion, $0+1$ vs. $2+3^{\text {b }}$ & $3.669(2.164-6.221)$ & $<0.0001^{\mathrm{a}}$ & $3.628(2.090-6.298)$ & $<0.0001^{\mathrm{a}}$ \\
\hline Venous invasion, $0+1$ vs. $2+3^{\mathrm{b}}$ & $1.859(1.137-3.038)$ & $0.0130^{\mathrm{a}}$ & & \\
\hline Perineural invasion, $0+1$ vs. $2+3^{\mathrm{b}}$ & $2.000(1.017-3.932)$ & $0.0450^{\mathrm{a}}$ & & \\
\hline B3GNT6 expression in cancer ${ }^{\mathrm{c}}$, -vs. + & $0.573(0.351-0.935)$ & $0.0260^{\mathrm{a}}$ & $0.496(0.300-0.818)$ & $0.0060^{\mathrm{a}}$ \\
\hline $\begin{array}{l}\text { MECA-79 antigen expression in cancer }{ }^{\mathrm{d}} \text {, } \\
\text { - vs. }+\end{array}$ & $2.245(1.285-3.923)$ & $0.0050^{\mathrm{a}}$ & & \\
\hline
\end{tabular}

B, Overall survival

Univariate analysis

Variables

Age, $\leq 72$ years vs. $>72$ years

Sex, female vs. male

Pathological tumor status, Tis+T1+T2 vs. T3

Pathological node status, N0 vs. N1+N2

Pathological metastatic status, M0 vs. M1

Histological grade, G1 vs. G2-4

Tumor margin status, negative vs. positive

Lymphatic invasion, $0+1$ vs. $2+3^{\mathrm{b}}$

Venous invasion, $0+1$ vs. $2+3^{\text {b }}$

Perineural invasion, $0+1$ vs. $2+3^{\mathrm{b}}$

B3GNT6 expression $^{c}$, - vs. +

MECA-79 antigen expression ${ }^{\mathrm{d}}$, -vs. +

\begin{tabular}{ccccc}
\hline HR $(95 \%$ CI $)$ & P-value & HR $(95 \%$ CI $)$ & P-value \\
\cline { 5 - 5 } $0.865(0.471-1.589)$ & 0.6410 & & \\
$0.472(0.225-0.992)$ & $0.0470^{\mathrm{a}}$ & & $0.0020^{\mathrm{a}}$ \\
$2.840(1.312-6.146)$ & $0.0080^{\mathrm{a}}$ & $3.753(1.606-8.766)$ & $0.0230^{\mathrm{a}}$ \\
$2.827(1.510-5.291)$ & $0.0010^{\mathrm{a}}$ & $2.179(1.116-4.254)$ & \\
$2.577(0.909-7.306)$ & 0.0750 & & $<0.0001^{\mathrm{a}}$ \\
$1.625(0.776-3.402)$ & 0.1980 & & \\
$2.345(1.284-4.283)$ & $0.0060^{\mathrm{a}}$ & & $0.0002^{\mathrm{a}}$ \\
$4.872(2.485-9.552)$ & $<0.0001^{\mathrm{a}}$ & $4.817(2.314-10.026)$ & \\
$3.010(1.642-5.518)$ & $0.0004^{\mathrm{a}}$ & & \\
$1.723(0.795-3.731)$ & 0.1680 & & \\
$0.442(0.240-0.817)$ & $0.0090^{\mathrm{a}}$ & $0.282(0.146-0.545)$ & \\
$2.574(1.279-5.181)$ & $0.0080^{\mathrm{a}}$ & &
\end{tabular}

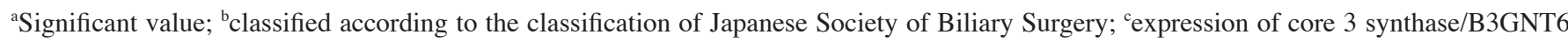
in non-invasive or invasive cancer cells; ${ }^{d}$ expression of 6-sulfated $\mathrm{N}$-acetyllactosamine on the extended core- $1 O$-glycans as indicated by MECA-79 antigen staining in invasive cancer cells. B3GNT6, $\beta 1,3-N$-acetylglucosaminyltransferase 6 ; +, positive; -, negative.

in OS and DFS, respectively; middle panels of Fig. 4). The dCCA patients with positive staining in the invasive region also showed a slightly good correlation with $\mathrm{OS}(\mathrm{P}=0.029)$ relative to those with negative $\mathrm{dCCA}$; however, not significantly correlated in DFS ( $\mathrm{P}=0.153)$ (right panels of Fig. 4). Moreover, there was no significant correlation between core 3 synthase expression and outcome in patients with pCCA (Fig. S2).

Various clinicopathological factors shown in Table II were investigated to determine whether they were prognostic. When the significant factors identified at univariate analysis were subjected to the multivariate analysis; core 3 synthase positive in cancer cells, no nodal metastasis, negative tumor margin status, and lower lymphatic invasion were closely associated with longer DFS. In addition, core 3 synthase positive in cancer cells, lower tumor status, no nodal metastasis and lower lymphatic invasion were significantly associated with longer OS. Thus, dCCA patients expressing core 3 synthase, regardless of the non-invasive or invasive region, displayed a significantly better prognosis than negative dCCA patients and it is indicated that core 3 synthase expression in any cancer cells is an independent prognosticator. As presented in Table SI, core 3 synthase displayed high positivity accounting for $100 \%$ of positive cases in tumors in situ or at the zero stage. 
MECA-79 expression correlates with a poor prognosis for $d C C A$ patients. MECA-79 positivity in the invasive cancer region of the dCCA was significantly associated with patient survival rate in an unfavorable manner $(\mathrm{P}=0.006$ for $\mathrm{OS}$, $\mathrm{P}=0.004$ for DFS) (right panels of Fig. 5). While MECA-79 positivity in the non-invasive cancer did not have any significant association with patient outcome (Fig. 5). When the significant factors identified at univariate analysis were subjected to the multivariate analysis, expression of MECA-79 antigen in invasive cancer cells was not a significant factor in the multivariate analyses of both DFS and OS (Table II). There was no significant survival effect of MECA-79 antigen expression in cancer cells found in pCCA (Fig. S3).

When the correlation between MECA-79 antigen expression and the various clinicopathological factors was analyzed, MECA-79 positivity was significantly associated with the node status, and cancer progression, such as lymphatic and venous invasion (Table SI).

Comparison of survival rates between the G8-144(+)MECA-79(-) and the G8-144-MECA-79(+) groups of patients with $e C C A$. From the total of 185 patients with eCCA, two groups, G8-144(+)-MECA-79(-) and G8-144(-)-MECA-79(+), were selected and analyzed to compare which $O$-glycan pathway (core 3 or core 1) was associated with a longer survival rate. From the results, we suggest that G8-144 is a favorable type of core structure in dCCA. The OS and DSF of G8-144 and MECA-79 in the eCCA cases are shown in Fig. 6. Both OS and DFS were longer in the G8-144(+)-MECA-79(-) group than in the G8-144(-)-MECA-79(+) group, except for the OS of the non-invasive eCCA cases. These results suggest that when eCCA with G8-144(+)-MECA-79(-) progresses to G8-144(+)-MECA-79(+) and G8-144(-)-MECA-79(+), the survival rate may decrease as malignant transformation progresses.

No association between the expression of core 3 synthase and MECA-79 in the CCA cells. We expected to find an inverse relationship between G8-144 and MECA-79 expression due to their glycan structures. However, there was no significant correlation between the expression of core 3 synthase and MECA-79 in the CCA cells (Table SII). This may be due to the fact that both antigens are not diffusely expressed on tumor cells in the same case, but are often expressed on some tumor cells separately.

The frequency of G8-144 expression is high in non-invasive intramucosal cancers and tends to decrease as the cancer cells invade deeper into the mucosa, but the low frequency of G8-positive tumor cells makes data generation difficult. However, based on the characteristics of G8-144, which is frequently expressed in non-invasive intramucosal cancers, the relationship between G8-144 and depth of invasion should be statistically significant. This would also support that G8-144 is a good prognostic marker, but it is unclear since we have not searched for it.

\section{Discussion}

The alteration of the $O$-glycan structures in cancer is one of the characteristics responsible for its aggressiveness $(17,25)$.
Core 3 expression has been reported to suppress tumor progression and inhibit metastasis in sarcoma, pancreatic, and prostate cancer cells $(10,12,26)$, while aberrant $O$-glycans, such as Tn and STn, are increased in various carcinomas $(17,27,28)$. Because antibodies recognizing the core 3 structure have not been established for diagnosis, we opted to use the antibody that recognizes core 3 synthase $(11,29,30)$.

In our previous study, we found that the expression of core 3 synthase was restricted in Goblet cells of the colon; however, its expression level was decreased during cancer progression and completely absent in colon cancer tissues $(10,11)$. In fact, the mRNA level of B3GNT6 was identified to be almost negative in more than 100 cancer cell lines examined by qRT-PCR and RNA-Seq (22) (data not shown). However, two studies ((24) and this study) demonstrated that some cancer tissues of PDAC and CCA expressed core 3 synthase. core 3 synthase was found to be expressed in approximately $20 \%$ of PDAC cases, and a higher expression of this enzyme was found in PDAC cells with more differentiated adenocarcinoma cases (24). In the present study, core 3 synthase was found to be positive in CCA and was mainly observed in the non-invasive area. Such findings indicate that core 3 synthase expression is induced in differentiated cancers of the pancreas and bile ducts. Importantly, the detection of core 3 synthase in patients with PDAC or dCCA was significantly associated with a longer survival rate than core 3 synthase-negative patients (24), Fig. 3). Such findings suggest that core 3 synthase can be a favorable prognostic marker for dCCA patient. Consistent with our results, the mRNA expression level of B3GNT6 was predicted to be a favorable marker for colorectal cancer (21). Thus, immunohistochemical detection of core 3 synthase would be useful to rapidly detect and diagnose several cancers that might secrete $O$-glycosylated proteins as their cellular characteristic.

In contrast to $\mathrm{dCCA}$, the prognosis of pCCA patients did not correlate with the expression of core 3 synthase (Fig. S2). Although both dCCA and pCCA are included in the eCCA, pCCA is located in the extrahepatic biliary tree near hepatic hilar area and easy to extend both right and left hepatic ducts. As a result, surgical operation is more difficult and causes higher unresectable rates (31) which lead to a poor survival rate. A previous report suggested that core 3 might be involved in the differentiation of colon tissues (10). Although the number of pCCA cases was fewer than that of dCCA, pCCA had a higher percentage of G8-144 positivity than dCCA. Thus, core 3 synthase may be differentially expressed in CCA cells according to the localization, which should be associated with bile duct differentiation. The effect of core 3 synthase expression on the survival rate of $\mathrm{dCCA}$ and $\mathrm{pCCA}$ was thus inconsistent.

As shown in Fig. 1, core 1 structures are synthesized at the 3' position of GalNAc on mucins, which is shared with core 3 structures. Core 1 synthase requires co-expression with the cofactor Cosmc (32), thus, the expression of core 1 synthase alone does not determine the core 1 structure. Because of this complexity, we did not stain the samples with an anti-core 1 synthase antibody. In addition, antibodies against the core 3 glycan structure are not yet available. Therefore, to analyze the differences between the expression of core 1 and core 3 in association with the prognosis of CCA, we used the 

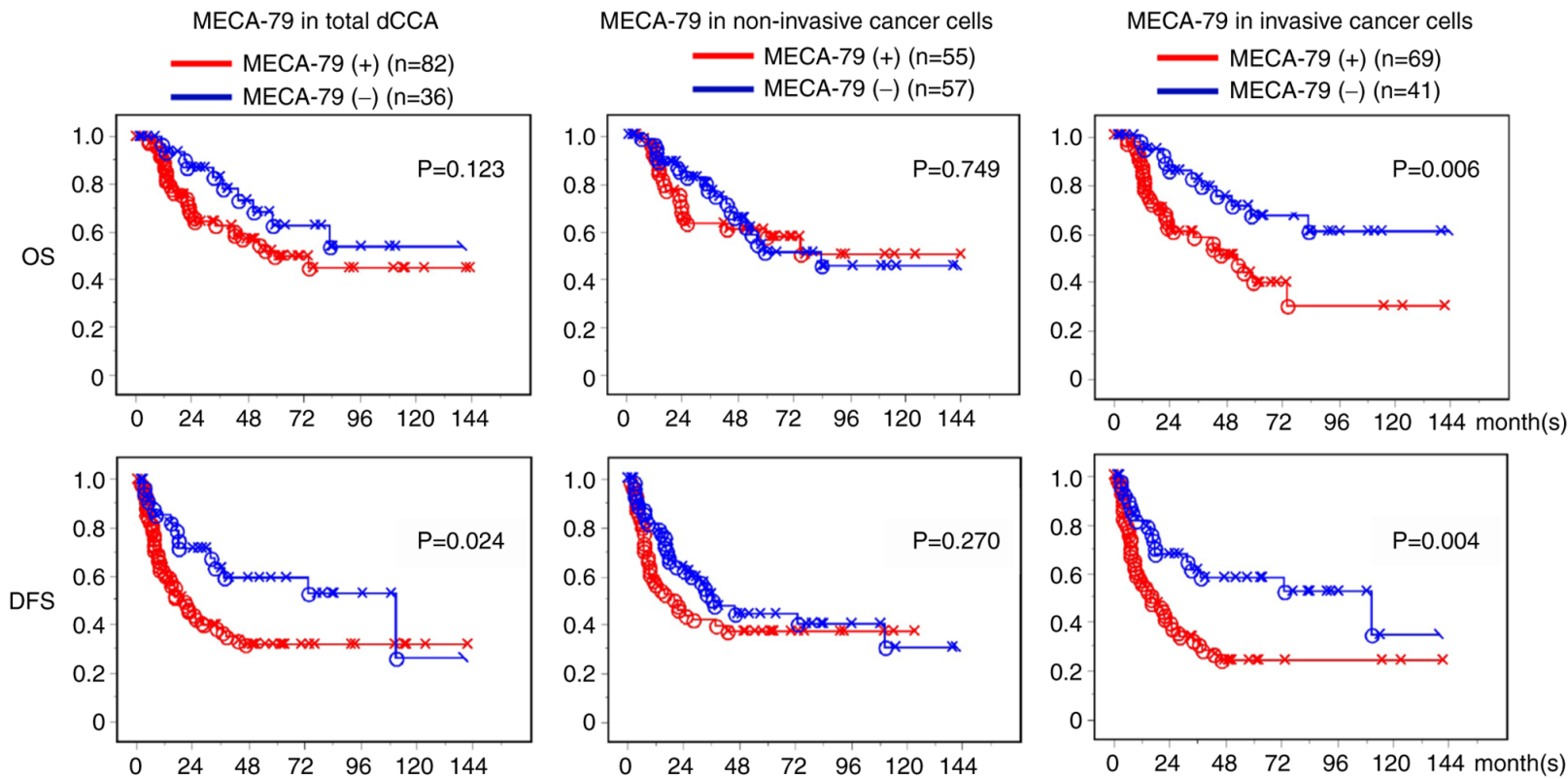

Figure 5. Survival rates of patients with dCCA with the MECA-79 epitope. Kaplan-Meier survival curves were plotted for patients with MECA-79(+) (red) or MECA-79(-) (blue) dCCA. Postoperative OS rates are presented in the upper panels and DFS rates are presented in the lower panels. Total dCCA (left panels), non-invasive cancer cells (center panels) and invasive cancer cells (right panels). The expression level of MECA-79 in the invasive area was significantly associated with worse survival of patients (right panels). dCCA, distal cholangiocarcinoma; OS, overall survival; DFS, disease-free survival; (+), positive; (-), negative.

Total eCCA
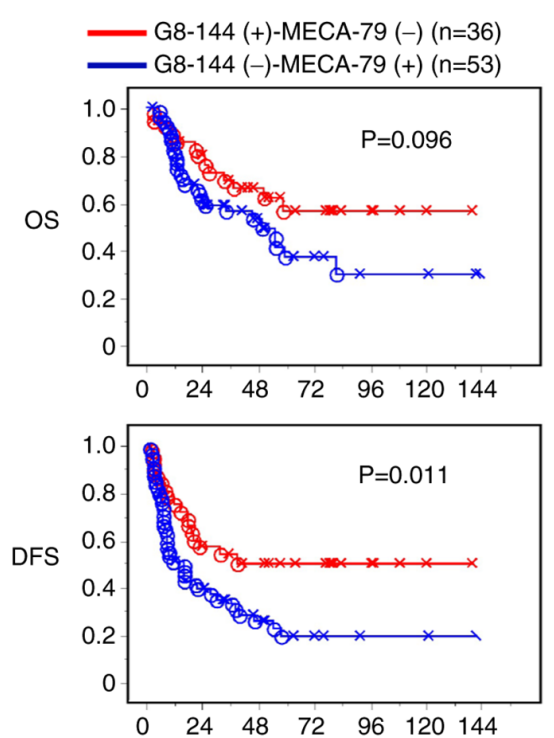

eCCA non-invasive
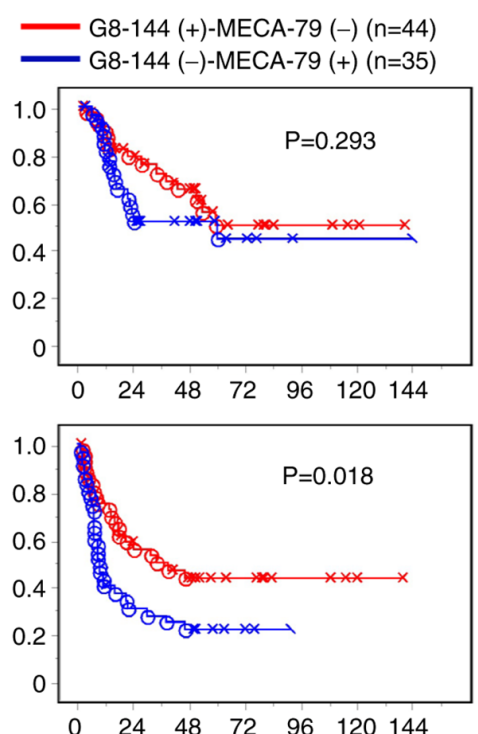

eCCA invasive cancer cells

G8-144 (+)-MECA-79 (-) (n=22)

G8-144 (-)-MECA-79 (+) $(n=61)$
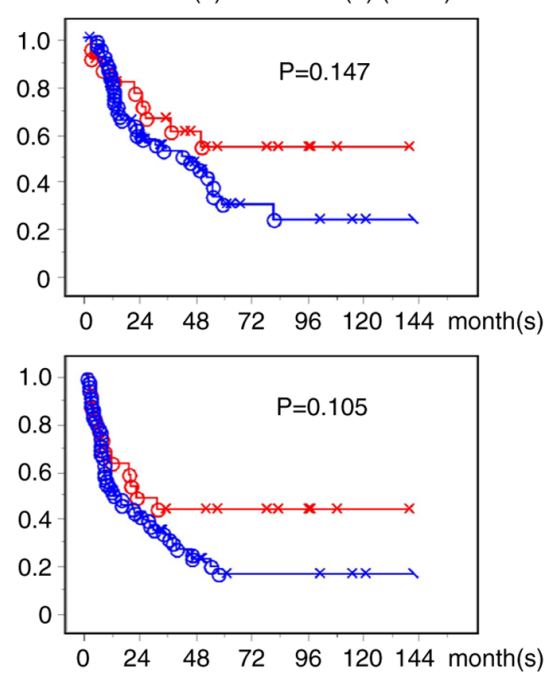

Figure 6. Survival rates of patients with eCCA. Kaplan-Meier survival curves were plotted for patients with G8-144(+)-MECA-79(-) (red) and G8-144(-)-MECA-79(+) (blue) eCCA. Compared with the expression level of MECA-79(+) with G8-144(-), the expression level of G8-144(+) with MECA-79(-) was significantly associated with the longer survival of patients after tumor resection. Postoperative OS rates are presented in the upper panels and DFS rates are presented in the lower panels. Total eCCA (left panels), non-invasive eCCA (center panels) and invasive eCCA (right panels). eCCA, extrahepatic cholangiocarcinoma; OS, overall survival; DFS, disease-free survival; (+), positive; (-), negative.

MECA-79 antibody and the G8-144 antibody as alternative tools for core 1 and core 3, respectively. As core 1 synthase is involved in the progression of cancer $(17,33)$, the invasive front of the cancer, which are located between cancer and normal tissues, displayed strong positivity of cancer cells with MECA-79, as shown in Fig. 2. Further, the MECA-79 epitope was identified to be increased upon cancer progression and is associated with poor prognosis in gastric cancers $(29,34)$. MECA-79 was thus identified to be associated with a shorter survival rate of dCCA, especially when expressed in invasive cancer areas (Fig. 5). Based on the statistical analyses, MECA-79 positivity was found to be significantly correlated with the cancer metastasis factors examined, as summarized in Table SI. Such findings suggest that MECA-79 is an unfavorable prognosticator for dCCA patients. Considering the synthetic pathway of the $O$-glycan cores, core 3 synthase may suppress MECA-79 expression in vivo. Indeed, a mirror-image stain was observed between G8-144 and MECA-79 in eCCA; 

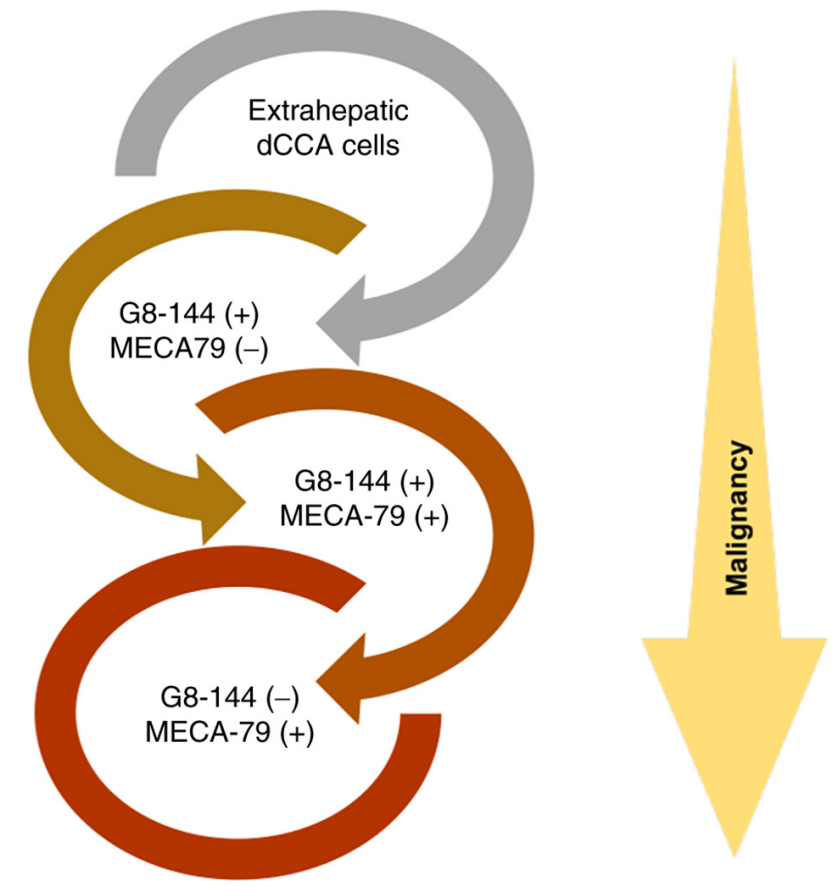

Figure 7. Schematic review of dCCA cancer cells with G8-144 and MECA-79 expression. Analysis of the expression patterns of G8-144 and MECA-79 in dCCA tissue and the survival rates of patients suggest that the malignancy of dCCA is increased when the expression of G8-144 is decreased, while MECA-79 expression is increased. dCCA, distal cholangiocarcinoma; $(+)$, positive; (-), negative.

core 3 synthase expressing mainly in intraepithelial or shallow invasive cancer cells and MECA-79 antigen expressing mainly in deeply invasive cancer cells, and rare event of cancer cells expressing both core 3 synthase and MECA-79 antigen (data not shown). According to the multivariate survival analyses (Table II), core 3 synthase expression in CCA cells was an independent prognosticator, although the association between the expression of core 3 synthase and MECA-79 in CCA cells was not determined. There are some limitations in this study as the data collection and analyses of our clinicopathological study were performed retrospectively. Using antibodies that directly detect core 3 or core 1 glycan structures might be better than detecting the enzymes themselves; however, the synthesis pathway and the glycan structures after core 3 are quite complicated, and no antibody specific against the core 3 structure is available.

In the present study, we aimed to identify prognostic biomarkers for patients with eCCA. We analyzed two candidate markers, core 3 synthase (G8-144) and core 1 containing glycan (MECA-79). Based on our findings, core 3 synthase was found to be expressed in non-invasive cancer cells. Further, it was implied that expression of core 3 synthase in cancer cells is an independent prognostic factor for dCCA patients predicting the better prognosis. However, a good correlation was not found with other cancer progression variables. The MECA-79 epitope was also observed to be increased in invasive cancer cells, and MECA-79 positivity correlated closely with the worse lifespan of dCCA patients. It has been confirmed that core 3 synthase is expressed in dCCA cells in situ. If the core 3 pathway competes with the core 1 pathway in vivo, then the core 1 pathway should become dominant as the expression level of the core 3 pathway decreases. As the expression level of core 3 synthase decreases, dCCA cells may turn into MECA-79-positive cells and become more malignant. Taken together, the observations of our staining experiments and statistical analysis suggest that the decrease in the Core 3 pathway expression (represented by G8-144) followed by an increase in the Core 1 pathway (represented by MECA-79) expression could be associated with the progression of dCCA (Fig. 7). These findings imply that these two antibodies could be employed to analyze the $O$-glycan pathways to predict the prognosis of patients with dCCA.

\section{Acknowledgements}

Not applicable.

\section{Funding}

This work was supported by the Japan Science and Technology Agency through the e-ASIA Joint Research Program (grant nos. JP18jm0210045 and 20ck0106532).

\section{Availability of data and materials}

The datasets used and/or analyzed during the current study are available from the corresponding author on reasonable request.

\section{Authors' contributions}

PB collected, analyzed and validated the data, and wrote the original manuscript draft. YI collected and analyzed the data, and provided resources. NH conceived and designed the methodology of the present study, collected, analyzed and validated the data, was responsible for providing the resources and acquiring funding, and reviewed and edited the manuscript. KS provided resources and performed data analysis of the patient clinical records. KA conceived and designed the methodology of this study, and wrote the original draft of the manuscript. HN conceived and designed this study, was responsible for acquiring funding, supervised the experiments, and reviewed and edited the manuscript. PB and NH confirm the authenticity of all the raw data. All authors have read and approved the final manuscript.

\section{Ethics approval and consent to participate}

This study was approved by the Institutional Review Board of the National Cancer Center (approval no. 2019-0186; Tokyo, Japan). Written informed consent was obtained from all participants before their participation in the study. All clinical investigations were conducted in accordance with the principles of the Declaration of Helsinki.

\section{Patient consent for publication}

Not applicable.

\section{Competing interests}

The authors declare that they have no competing interests. 


\section{References}

1. Cancer International Agency for Research on Cancer: Cancer fact sheets. In: Cancer Today International Agency for Research on Cancer, World Health Organization, 2020.

2. Nagtegaal ID, Odze RD and Klimstra D: WHO Classification of Tumours, Digestive System. International Agency for Research on Cancer, Wiley-Blackwell, Lyon, 2019.

3. Amin MB, Edge S, Greene F, Byrd DR, Brookland RK, Washington MK, Gershenwald JE, Compton CC, Hess KR, Sullivan DC, et al: AJCC Cancer Staging Manual. Springer International Publishing, New York, NY, 2017.

4. Brierley JD, Gospodarowicz MK and Wittekind C (eds) Union for International Cancer Control: TNM Classification of Malignant Tumours. John Wiley \& Sons, Inc., Oxford, UK, 2017

5. Kamsa-Ard S, Luvira V, Suwanrungruang K, Kamsa-Ard S, Luvira V, Santong C, Srisuk T, Pugkhem A, Bhudhisawasdi V and Pairojkul C: Cholangiocarcinoma trends, incidence, and relative survival in khon kaen, thailand from 1989 through 2013: A population-based cancer registry study. J Epidemiol 29: 197-204, 2019

6. Banales JM, Marin JJG, Lamarca A, Rodrigues PM, Khan SA, Roberts LR, Cardinale V, Carpino G, Andersen JB, Braconi C, et al: Cholangiocarcinoma 2020: The next horizon in mechanisms and management. Nat Rev Gastroenterol Hepatol 17: $557-588,2020$

7. Dodson RM, Weiss MJ, Cosgrove D, Herman JM, Kamel I, Anders R, Geschwind JF and Pawlik TM: Intrahepatic cholangiocarcinoma: Management options and emerging therapies. J Am Coll Surg 217: 736-750.e4, 2013

8. Stowell SR, Ju T and Cummings RD: Protein glycosylation in cancer. Ann Rev Pathol 10: 473-510, 2015.

9. Varki A, Cummings RD, Esko JD, Stanley P, Hart GW, Aebi M, Darvill AG, Kinoshita T, Packer NH, Prestegard JH, et al: Essentials of Glycobiology. 3rd edition. Cold Spring Harbor Laboratory Press, Cold Spring Harbor, NY, 2015-2017.

10. Iwai T, Kudo T, Kawamoto R, Kubota T, Togayachi A, Hiruma T, Okada T, Kawamoto T, Morozumi K and Narimatsu H: Core 3 synthase is down-regulated in colon carcinoma and profoundly suppresses the metastatic potential of carcinoma cells. Proc Natl Acad Sci USA 102: 4572-4457, 2005.

11. Iwai T, Inaba N, Naundorf A, Zhang Y, Gotoh M, Iwasaki H, Kudo T, Togayachi A, Ishizuka Y, Nakanishi $\mathrm{H}$ and Narimatsu $\mathrm{H}$ : Molecular cloning and characterization of a novel UDP-GlcNAc: GalNAc-peptide beta1,3-N Acetylglucosaminyltransferase (beta 3Gn-T6), an Enzyme Synthesizing the Core 3 Structure of O-Glycans. J Biol Chem 277: 12802-12809, 2002.

12. Lee SH, Hatakeyama S, Yu SY, Bao X, Ohyama C, Khoo KH, Fukuda MN and Fukuda M: Core3 O-Glycan synthase suppresses tumor formation and metastasis of prostate carcinoma PC3 and LNCaP cells through Down-regulation of alha2bata1 Integrin Complex. J Biol Chem 284: 17157-17169, 2009.

13. Ye J,Wei X, Shang Y,Pan Q, Yang M,Tian Y,He Y,Peng Z, Chen L, Chen W and Wang R: Core 3 mucin-type O-glycan restoration in colorectal cancer cells promotes MUC1/p53/miR-200c-dependent epithelial identity. Oncogene 36: 6391-6407, 2017.

14. Pearce OMT: Cancer glycan epitopes: Biosynthesis, structure and function. Glycobiology 28: 670-696, 2018.

15. Springer GF, Desai PR and Banatwala I: Blood group MN specific substances and precursors in normal and malignant human breast tissues. Naturwissenschaften 61: 457-458, 1974.

16. Springer GF: $T$ and $T n$, general carcinoma autoantigens. Science 224: 1198-1206, 1984.

17. Chia J, Goh G and Bard F: Short O-GalNAc glycans: Regulation and role in tumor development and clinical perspectives. Biochim Biophys Acta 1860: 1623-1639, 2016.

18. Hagisawa S, Ohyama C, Takahashi T, Endoh M, Moriya T, Nakayama J, Arai Y and Fukuda M: Expression of core 2 beta1, 6-N-acetylglucosaminyltransferase facilitates prostate cancer progression. Glycobiology 15: 1016-1024, 2005.
19. Brockhausen I: Mucin-type O-glycans in human colon and breast cancer: Glycodynamics and functions. EMBO Rep 7: 599-604, 2006.

20. Yeh JC, Hiraoka N, Petryniak B, Nakayama J, Ellies LG, Rabuka D, Hindsgaul O, Marth JD, Lowe JB and Fukuda M: Novel sulfated lymphocyte homing receptors and their control by a core 1 extension beta 1,3-N-Acetylglucosaminyltransferase. Cell 105: 957-969, 2001

21. Proteomics. Tissue-based map of the human proteome. Science 347: 1260419, 2015. https://www.proteinatlas.org/ ENSG00000198488-B3GNT6. Accessed July 23, 2021.

22. Angata K, Sawaki H, Tsujikawa S, Ocho M, Togayachi A and Narimatsu H: Glycogene expression profiling of hepatic cells by RNA-Seq analysis for Glyco-biomarker identification. Front Oncol 10: 1224, 2020.

23. Ino Y, Yamazaki-Itoh R, Oguro S, Shimada K, Kosuge T, Zavada J, Kanai Y and Hiraoka N: Arginase II expressed in cancer-associated fibroblasts indicates tissue hypoxia and predicts poor outcome in patients with pancreatic cancer. PLoS One 8: e55146, 2013.

24. Doi N, Ino Y, Angata K, Shimada K, Narimatsu H and Hiraoka N: Clinicopathological significance of core $3 \mathrm{O}$-glycan synthetic enzyme, $\beta 1,3-\mathrm{N}$-acetylglucosaminyltransferase 6 in pancreatic ductal adenocarcinoma. PLoS One 15: e0242851, 2020.

25. Pinho SS and Reis CA: Glycosylation in cancer: Mechanisms and clinical implications. Nat Rev Cancer 15: 540-555, 2015.

26. Radhakrishnan P, Grandgenett PM, Mohr AM, Bunt SK, Yu F, Chowdhury S and Hollingsworth MA: Expression of core 3 synthase in human pancreatic cancer cells suppresses tumor growth and metastasis. Int J Cancer 133: 2824-2833, 2013.

27. Fu C, Zhao H, Wang Y, Cai H, Xiao Y, Zeng Y and Chen $\mathrm{H}$ : Tumor-associated antigens: Tn antigen, sTn antigen, and T antigen. HLA 88: 275-286, 2016.

28. Ju T, Wang Y, Aryal RP, Lehoux SD, Ding X, Kudelka MR, Cutler C, Zeng J, Wang J, Sun X, et al: Tn and sialyl-Tn antigens, aberrant $\mathrm{O}$-glycomics as human disease markers. Proteomics Clin Appl 7: 618-631, 2013.

29. Okayama H, Kumamoto K, Saitou K, Hayase S, Kofunato Y, Sato Y, Miyamoto K, Nakamura I, Ohki S, Koyama Y, et al: Ectopic expression of MECA-79 as a novel prognostic indicator in gastric cancer. Cancer Sci 102: 1088-1094, 2011.

30. Steentoft C, Yang Z, Wang S, Ju T, Vester-Christensen MB Festari MF, King SL, Moremen K, Larsen ISB, Goth CK, et al: A validated collection of mouse monoclonal antibodies to human glycosyltransferases functioning in mucin-type O-glycosylation. Glycobiology 29: 645-656, 2019.

31. Dondossola D, Ghidini M, Grossi F, Rossi G and Foschi D: Practical review for diagnosis and clinical management of perihilar cholangiocarcinoma. World J Gastroenterol 26: 3542-3561, 2020.

32. Aryal RP, Ju T and Cummings RD: The endoplasmic reticulum chaperone cosmc directly promotes in vitro folding of T-synthase. J Biol Chem 285: 2456-2462, 2010.

33. Gupta R, Leon F, Rauth S, Batra SK and Ponnusamy MP: A systematic review on the implications of O-linked glycan branching and truncating enzymes on cancer progression and metastasis. Cells 9: 446, 2020.

34. Hoshino H, Ohta M, Ito M, Uchimura K, Sakai Y, Uehara T, Low S, Fukushima $M$ and Kobayashi M: Apical membrane expression of distinct sulfated glycans represents a novel marker of cholangiolocellular carcinoma. Lab Invest 96: 1246-1255, 2016.

This work is licensed under a Creative Commons Attribution-NonCommercial-NoDerivatives 4.0 International (CC BY-NC-ND 4.0) License. 